\title{
Environmental Impact of Coal Clay Brick Kiln Emission on the Reproductive Health of Children and Adult Male Workers
}

\author{
Saadia Shahid, Adila Zafar, and Sarwat Jahan
}

\begin{abstract}
The emission from the brick kilns is a major cause of air pollution worldwide, specifically in developing countries. These pollutants can have a direct impact on reproductive health of adult males and children working in brick kilns, and can eventually lead to male infertility. The aim of this research was to check the effect of brick kiln exposure on the serum testosterone, luteinizing hormone ( $\mathrm{LH})$, and body mass index (BMI) of male children and adults working in kilns. Total 205 subjects, constituting, 54 exposed children $(n=54)$ and exposed male adults $(n=58)$ working in brick kilns were analyzed against healthy children $(n=48)$ and healthy males $(n=45)$ from Rawalpindi, Pakistan. The age range of children under study was 4-17 years and of adults was 18-48 years, further subdivided into sub groups. In case of children $(n=54)$ our findings indicated that the brick kiln exposure significantly reduces the level of $\mathrm{LH}(P<0.01)$, testosterone $(P<0.5)$ and $\mathrm{BMI}$ $(P=0.01)$. Similarly, adult brick kilns workers $(n=58)$ when analyzed and compared with control $(n=45)$ indicated significantly lower $B M I$ values $(P<0.01)$ and reduced levels of serum testosterone and $\mathrm{LH}$ levels $(P<0.05)$. Our results are in accordance with our hypothesis that coal clay brick kiln exposure can directly affect the fertility of children and adult males.
\end{abstract}

Index Terms-Brick kilns, body mass index, children, coal clay, exposure, luteinizing hormone, males, reproductive health, testosterone.

\section{INTRODUCTION}

The fact that excessive occupational exposure can lead drastically to male reproductive malfunctioning was considered with a very first publication in 1970s which suggested that lead might be cause of impaired reproductive function [1]. Since, then various authors have long-established the possible significant drop in sperm quality and increase in male infertility as a consequence of occupational exposure [2], [3].

Traditional Brick Kiln industry fueled by coal [4] is a source of hazardous emission, composed of fine dust

Manuscript received September 22, 2016; revised February 24, 2017. The study gives an insight into the havocs of prolonged occupational exposure especially for vulnerable populations. Occupational exposure assessment in relevance to reproductive health of children and adults males working in coal clay brick kilns, commonly found in developing countries i.e. Pakistan, Bangladesh, India (South Asia).Quantitative enzyme immunoassay (ELISA) run on the blood samples of male children and adults showed a low level of testosterone and LH as compared to healthy males which is an indication towards drastic occupational exposure affects that needs to be catered through insightful research that leads to solutions.

The authors are with the Animal Sciences, Quaid-i-Azam University Islamabad, Pakistan (e-mail: saadiashahidrafiq@yahoo.com, adilazaffar@ymail.com,sjahan@qau.edu.pk). particles, Hydrocarbons, Sulphur Dioxide (SO2), Oxides of Nitrogen (NOx), Fluoride compounds, Carbon Monoxide (CO), Small amount of carcinogenic dioxins, Aldehydes and Free radicals [5], resulting in reproductive health problems in male brick kiln workers of all ages including children and adults, who are bound to work for prolonged periods [6]. Endocrine-disrupting chemicals (EDCs) found in emission can effect functionality of endocrine glands, such as the thyroid or pituitary which in turn can have adverse effects on the secretion of reproductive hormones of male workers [7]-[9]. Children working at brick kilns besides having poor health [10], have been reported to have the reproductive health issues including morphological alterations of spermatozoa [11], reduction of sperm density and motility [12], affect on semen volume and on fertility [13]. The hazardous pollutions and prolonged heath exposure [14] have also altered the LH and testosterone secretion in children [15] and adult male workers of Brick kilns [16].

Previous studies have reported adverse effects of brick kiln emission constituents such as lead, mercury, cadmium, nitrous oxide and hydrocarbons are capable of harming the reproductive health of adult males by damaging sperm directly [17].The brick kiln emission constituents including dioxins and other heavy metals can lead to hormonal disruption, decreased sperm count, descensus testis, altered male sexual behavior and reduced infertility in men [18]. Other constituents like heavy metals e.g. lead, cadmium mercury and persistent organic pollutants i.e., hydrocarbons found in brick kiln emission can cause morphological and functional gonadal dysfunction e.g. infertility and decreased libido, as a consequence of altered LH and testosterone secretions [19].

The major focus of the carried out research study was to evaluate the environmental impact of coal clay exposure on the general and reproductive health of male children and adults working at brick kilns for prolonged periods. The present study was set out to determine the BMI and extent of LH and Testosterone reduction in children and adult males working at brick kilns. The hormonal analysis carried out for measuring the LH and testosterone level gave an insight into reproductive health of the workers. The parameters like weight and height were considered for BMI comparison.

\section{MATERIAL AND METHODS}

\section{A. Subject and Sampling}

The coal clay brick kilns located at Rawat near Rawalpindi, Pakistan, were visited for subject sampling. The study criteria 
involved a personal interview, questionnaire and blood sample collection. The parameters such as weight, height, and smoke exposure were also considered. About 54 blood samples of exposed children $(n=54)$ aged (4-17 years) and 58 blood samples of adult males $(n=58)$ aged (18-48) were taken. The subjects working for more than 2 years in brick kilns were considered. For analysis and comparison 48 healthy children (aged 4-17 years) were selected from the public school. The samples of healthy males (control group $n=45$ ) were taken from university and surroundings.

\section{B. Experimental Design}

The child subjects were divided according to their pubertal stages into three groups. The three groups of child subjects were 4-8 years (pre-pubertal), 9-13 years (early pubertal) and 14-17 years (pubertal group). The allocated age groups of adult male subjects were 18-24 years, 25-29 years, 30-34 years and $45-48$ years.

\section{Blood Sample Collection}

After taking the required information from each subject, blood samples were taken from cephalic vein into $3 \mathrm{~mL}$ syringes by a trained medical technologist. Heparin was used as a anticoagulant. Blood samples collected in gel vacutainers was centrifuged at $3000 \mathrm{rpm}$ for $3 \mathrm{~min}$ for serum extraction. The Serum was transferred to eppendorf tubes and stored at $-20 \mathrm{C}$ in the deep freezer prior to analysis.

\section{Determination of $B M I$}

BMI of individual subjects was calculated using following formula:

$$
\begin{aligned}
\mathrm{BMI}= & (\text { Weight in Pounds } /(\text { Height in inches } \\
& \times \text { Height in inches })) \times 703 .
\end{aligned}
$$

The comparison of BMI values of controls verses exposed subjects was carried out to for final results.

\section{E. Determination of Serum $\mathrm{LH}$}

Quantitative enzyme immunoassay (ELISA) was performed by using commercially available kits obtained from Amegnix (USA) to determine the serum LH concentration.

\section{F. Determination of Serum Testosterone}

Quantitative enzyme immunoassay (ELISA) was performed by using commercially available kits obtained from Amegnix (USA) to determine the serum testosterone concentration.

\section{Figures AND TABLES}

TABLE I: DAILY EXPOSURE IN HOURS OF MALE CHILDREN AND ADULTS WORKING AT BRICK KILN

\begin{tabular}{llll}
\hline \hline $\begin{array}{l}\text { Duration of } \\
\text { Exposure }\end{array}$ & $8-10$ hours & $12-16$ hours & $16+$ hours \\
\hline Male children & $43 \%$ & $42 \%$ & $15 \%$ \\
Adults & $28 \%$ & $55 \%$ & $17 \%$ \\
\hline \hline
\end{tabular}

Values are expressed as mean \pm SEM. $\mathrm{P}<0.01$ shows the significant difference.
TABLE II: BMI EVALUATION IN CONTROL AND BRICK KILN MALE CHILD AND ADULT WORKERS. VALUES ARE EXPRESSED AS MEAN \pm SEM

\begin{tabular}{llll}
\hline \hline & Age groups & Control & Exposed \\
\hline Male children & Pre-pubertal & $19.62 \pm 0.67$ & $14.35 \pm 0.78$ \\
Male children & Early pubertal & $20.07 \pm 0.28$ & $15.29 \pm 0.5$ \\
Male children & Pubertal & $20.77 \pm 0.88$ & $19.72 \pm 0.72$ \\
Adults & $18-24$ & $22.25 \pm 0.69$ & $18.48 \pm 0.2$ \\
Adults & $25-29$ & $21.36 \pm 0.63$ & $20.22 \pm 0.4$ \\
Adults & $30-34$ & $22.1 \pm 0.48$ & $19.5 \pm 0.3$ \\
Adults & $35-48$ & $23.6 \pm 1.04$ & $18.71 \pm 0.46$ \\
\hline \hline
\end{tabular}

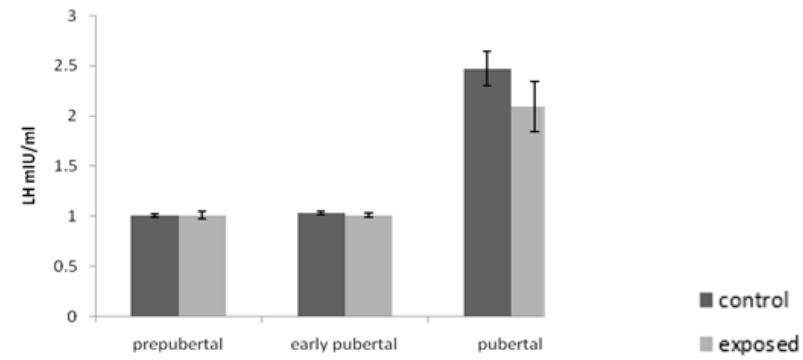

Fig. 1. Serum LH levels (mIU/ml) in control and exposed children working at brick kilns.

Values are expressed as mean \pm SEM. $P<0.05$ shows the significant difference.

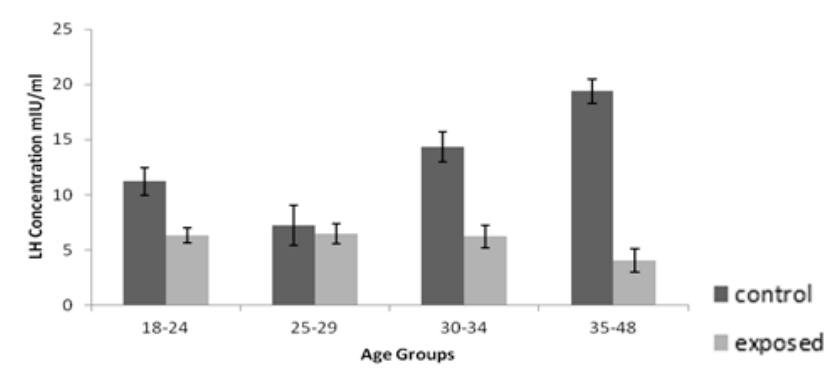

Fig. 2. Serum LH level in the adult males working at brick kilns.

Values are expressed as mean \pm SEM. $P<0.5$ shows the significant difference.

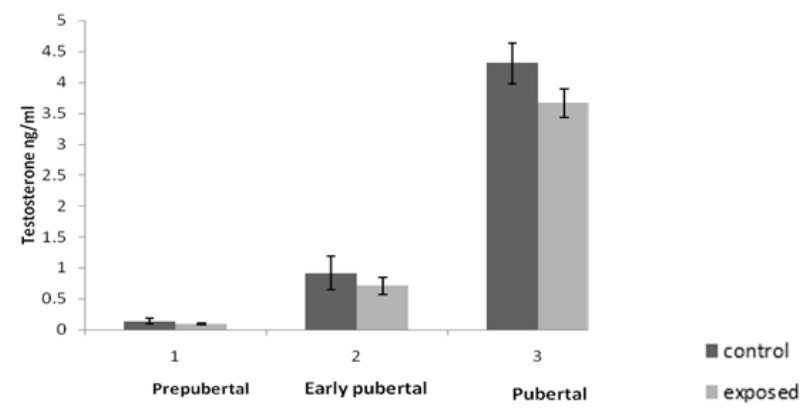

Fig. 3. Serum testosterone levels $(\mathrm{ng} / \mathrm{ml})$ in control and exposed children working at brick kilns.

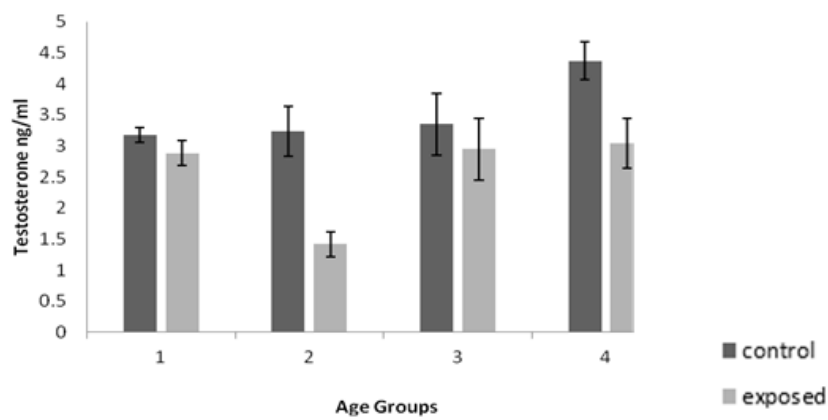

Fig. 4. Serum Testosterone level in the adult males working at brick kilns. 
Values are expressed as mean \pm SEM. $P<0.05$ shows the significant difference.

\section{Statistical Analysis}

The results were compared through one way of analysis of variance (ANOVA) using SPSS software (version 13.0). One way analysis of variance (single factor) and t-test analysis revealed the variation of different parameters which included body mass index, testosterone concentration and luteinizing hormone $(\mathrm{LH})$ concentration among different treated group of samples. In statistical analysis probability $\mathrm{P}$ value less than $0.05,0.01$ and 0.5 were considered different depending upon the taken parameters [20].

\section{RESUlts}

\section{A. Age}

The control and exposed samples of children were divided into three groups. Pre-pubertal group aged (4-8 years), early pubertal group aged (9-13 years) and pubertal group age (14-17 years). The percentage of children exposed to brick kiln heat and smoke in pre pubertal group was 30\%, early pubertal were $62 \%$ and pubertal were $8 \%$.

The control and exposed samples of adults were divided into four groups with respect to their ages. The age groups were 18-24 years, 25-29 years, 30-34 years and 35-48 years. The percentage of adult males exposed to brick kiln heat and smoke in age range 18-24 years was 45\%, 25-29 years was $15 \%, 30-34$ years was $15 \%$ and $35-48$ years was $25 \%$ (Table I).

\section{B. Work Duration}

The work duration of children working at brick kilns varied from individual to individual. $23 \%$ children were working for $7 \mathrm{hrs}$ per day, $22 \%$ for $12 \mathrm{hrs}$ and $8 \%$ children were working for $20 \mathrm{hrs}$ per day as they were considered as strong an could perform the hard task at these kilns (Table 1).

The work duration of males working at brick kilns varied from individual to individual. $19 \%$ of the adult male workers were working for 8 hours per day, $9 \%$ for 10 hours per day, $37 \%$ for 12 hours per day, $18 \%$ for 16 hours per day and $17 \%$ males were working for 24 hours per day (Table 1).

\section{BMI (Body Mass Index)}

\section{1) Control group}

BMI values were observed near to normal levels in healthy male children and adults.

\section{2) Exposed group}

A remarkable decrease $(P<0.01)$ was observed in the BMI values of pre-pubertal group (4-8 years) of children working at brick kilns as they are severely underweight when compared with the control group. BMI values were also significantly lower $(P<0.01)$ in early pubertal group as they were not in normal range in comparison to the control group. A significant reduction $(P<0.01)$ of BMI values was noticed in pubertal group of children working at brick kilns when compared to the control group (Table II).

A remarkable decrease $(P<0.01)$ was observed in the BMI values of adult males working at brick kilns as they are severely underweight when compared with the control group. All the age groups 18-24 years, 25-29 years, 30-34 years and $35-48$ years were equally affected (Table II).

\section{Hormonal Assays (Leuteinizing Hormone (LH))}

\section{1) Control group}

In the healthy control group of male children and adult males, LH levels were considered normal.

\section{2) Exposed group}

LH levels were significantly decreased $(P<0.01)$ in pre-pubertal group in comparison to the control group. A remarkable decrease $(P<0.01)$ was observed in the serum LH levels of early pubertal group of children working at brick kilns when compared with the control group. A significant reduction $(P<0.01)$ of $\mathrm{LH}$ level was noticed in pubertal group of children working at brick kilns then the control group (Fig. 1).

LH levels were decreased $(P<0.05)$ in adult males falling all age groups, when compared to the control groups. LH levels were significantly decreased $(P<0.05)$ in adult males falling in all exposed subjects in comparison to the control subjects. All the age groups 18-24 years, 25-29 years, 30-34 years and 35-48 years were equally affected (Fig. 2).

\section{E. Testosterone}

\section{1) Control group}

Testosterone levels were observed near to normal levels in healthy male children and male adults.

\section{2) Exposed group}

A remarkable decrease $(P<0.5)$ was observed in the serum testosterone levels of pre-pubertal group of children working at brick kilns when compared with the control group. Testosterone levels were significantly decreased $(P<0.5)$ in early pubertal group in comparison to the control group. A significant reduction $(P<0.5)$ was noticed in pubertal group of children working at brick kilns then the control group (Fig. 3).

In male adults a remarkable decrease $(P<0.05)$ in the serum testosterone levels was observed in exposed subjects when compared to the control groups. Subjects of all exposed groups i.e. 18-24 years, 25-29 years, 30-34 years and 35-48 years were affected. The adult males falling in age group 25-29 were affected most (Fig. 4).

\section{DISCUSSION}

The results of our present study indicate lowered concentrations of serum LH, serum testosterone and BMI values in children and adults working in a polluted environment of the brick kilns, as compared to controls. The poor socio-economic condition of the brick kiln workers can also be one strong factor for poor general and reproductive health [21]. These findings are in agreement with the previous findings of Woodruff which stated, occupational and residual exposure in relation to socio economic status may also be associated with air pollution levels and can influence reproductive outcomes [22].

Our findings verify that low BMI values of children and 
adults indicate overall poor health conditions of workers with poor immune system, making them prone to allergies, viral diseases, musculoskeletal problems, hypertension [8], respiratory issues, cardiac diseases [23] and skeleton deformities [5] with the passing time and excessive exposure.

However, the incidence of reproductive dysfunction did not vary across BMI categories, when evaluated for potential contributing factors, indicating need for further study. The exact mechanism of BMI-mediated effects on serum testosterone, sex hormone-binding globulin, $\mathrm{LH}$ and inhibin $\mathrm{B}$ is not fully understood [24].

The present study illustrates that time duration of exposed subjects was quite protracted. Prolonged hours of exposure can lead to multiple drastic effects on general and reproductive health of male children and adult brick kiln workers. These findings were in correspondence with work done by [25] establishing the facts that work duration is an important factor that formulates links between environmental health and reproductive problems. Occupational exposure to high temperature in several industries can lead to a higher prevalence of pathologic sperm profiles among the exposed subjects when compared to control subjects [26]. Excessive heat exposure for extended periods can have adverse effects on spermatogenesis and hormonal secretion eventually leading to male infertility [27].

The present investigation indicated that the male children and adults working at brick kilns had reduced levels of testosterone $(P<0.05)$ and $\mathrm{LH}(P<0.05)$ as compared to controls. These results directly match the findings of the study that the harmful environmental pollutants may affect the release of gonadotropins (FSH and $\mathrm{LH}$ ) and prolactin within the anterior hypophysis, as well as alter the feedback mechanism of the hypothalamus-hypophysis-gonads, and may have an impact on testosterone and inhibin B production or secretion in the testes [28]. These findings indicate that the toxicants emitted from brick kiln workers can have an everlasting effect on the reproductive health of adult workers. The impact of paternal exposure on progeny outcome includes, low birth weight, cancers, developmental, neuroendocrine, neurochemical abnormalities and congenital malformations [29]. The chances of primary and secondary infertility are higher in males exposed to different pharmacologic, chemical and physical agents [27]. These findings were in agreement with recent data of [25] that indicated the substances of concern, included elements like dioxins, PCB, heavy metals and estrogen mimicking toxicants, can derail human reproduction and development by effecting hormone secretion. The endocrine disrupting chemicals, which are probable constituents of brick kiln emission, induced a significant decrease in testosterone levels on exposure in male children and adults. The effects of some specific EDC include cryptorchidism, reduced leydig function, hypospadias, testis cancer, hormonal dysfunction, micropenis, reduced anogenital distance, delayed sexual maturation, reduced fertility and infertility [30].

\section{CONCLUSION}

Currently in Pakistan no significant study on reproductive health consequences of male children and adults working in brick kilns has been reported. The decreased level of serum $\mathrm{LH}$, testosterone and BMI values as indicated by the findings of current study imply that there is a need of extensive research to evaluate the reproductive health of brick kiln workers of Pakistan and other developing countries. Additionally, potential occupational human exposure, evocative evidence of probable deleterious effects on the developing reproductive system and sensitivity of the reproductive organs suggests that, there are significant grounds to be concerned about the emission from several industries, especially brick kilns [27]. Improvement of workplace environment, follow up of international criteria for industrial set up, Implementation of environmental laws, high grade fuel consumption and upgraded socio-economic status of brick kiln workers is the need of time [31]. By improving these conditions the current health status of brick kiln workers of Pakistan and other developing countries can be improved.

\section{ACKNOWLEDGMENT}

This research was conducted at Animal Sciences Department of Quaid-i-Azam University, Islamabad. The authors would like to thanks Dr. Serwat Jahan (Chairman Animal Sciences) for providing necessary facility for the successful completion of the research job.

\section{REFERENCES}

[1] L. Ioana et al., "Reproductive ability of workmen occupationally exposed to lead," Archives of Environmental Health: An International Journal, vol. 30, no. 8, pp. 396-401, 1975.

[2] C. M. Nelson and R. G. Bunge, "Semen analysis: Evidence for changing parameters of male fertility potential," Fertility and sterility, vol. 25 , no. 6 , pp. 503-507, 1974.

[3] S. Rémy et al., "Meeting report: Atmospheric pollution and human reproduction," Environmental Health Perspectives, vol. 116, no. 6, pp. 791-8, 2008

[4] M. Sameer et al., Energy Conservation and Pollution Control in Brick Kilns, Tata Energy Research Institute, Habitat Place, New Delhi, India, 1999.

[5] Erbe and S. Oliver, "Technical, economical and organizational analysis of informal brick production in Tercera Chica," Doctoral Diss. Cologne University of Applied Sciences, México, SLP, 2011.

[6] B1. Allen et al., "The benefits and costs of informal sector pollution control: Mexican brick kilns," Environment and Development Economics, vol. 11, no. 5, pp. 603-627, 2006.

[7] K. J. Robert et al., Research needs for the risk assessment of health and environmental effects of endocrine disruptors: A report of the US EPA-sponsored workshop," Environmental Health Perspectives, vol. 104, suppl. 4, 1996, p. 715

[8] Q. Erika et al., "Occupational exposure and effects on the male reproductive system," Cadernos de Saúde Pública, vol. 22, no. 3, pp. 485-493, 2006.

[9] T. J. Woodruff et al., Environmental Impacts on Reproductive Health and Fertility, Cambridge University Press, 2010.

[10] M. Tim et al., "Trends in work-related musculoskeletal disorder reports by year, type, and industrial sector: A capture-recapture analysis," American Journal of Industrial Medicine, vol. 48, no. 1, pp. 40-49, 2005.

[11] O. Kemal et al., "Semen quality of smoking and non-smoking men in infertile couples in a Turkish population," Archives of gynecology and obstetrics, vol. 271, no. 2, pp. 109-112, 2005.

[12] M. J. Zinaman et al., "Semen quality and human fertility: A prospective study with healthy couples," Journal of Andrology, vol. 21, no. 1, pp. 145-153, 2000.

[13] S. G. Selevan et al., "Semen quality and reproductive health of young Czech men exposed to seasonal air pollution," Environmental Health Perspectives, vol. 108, no. 9, pp. 887, 2000.

[14] Joffe and Michael, "Infertility and environmental pollutants," British Medical Bulletin, vol. 68, no. 1, pp. 47-70, 2003. 
[15] T. P. Ng et al., "Male endocrine functions in workers with moderate exposure to lead," British Journal of Industrial Medicine, vol. 48, no. 7, pp. 485-491, 1991.

[16] T. Spomenka et al., "Semen quality and reproductive endocrine function in relation to biomarkers of lead, cadmium, zinc, and copper in men," Environmental Health Perspectives, vol. 108, no. 1, pp. 45, 2000.

[17] R. Linda and M. R. Culler, "Clinical occupational medicine," Journal of Occupational and Environmental Medicine, vol. 28, no. 11, pp. 1138-1140, 1986.

[18] M. P. Waalkes, M. R. Anver, and B. A. Diwan, "Chronic toxic and carcinogenic effects of oral cadmium in the Noble $(\mathrm{NBL} / \mathrm{Cr})$ rat: Induction of neoplastic and proliferative lesions of the adrenal, kidney, prostate, and testes," Journal of Toxicology and Environmental Health Part A, vol. 58, no. 4, pp. 199-214, 1999.

[19] Waissmann and William, "Health surveillance and endocrine disruptors," Cadernos de Saúde Pública, vol. 18, no. 2, pp. 511-517, 2002.

[20] Berry PG: Statistical Methods in Medical Research, Blackwell Scientific Publications, Oxford and Edinburgh UK 1985.

[21] B. Silvia and A. M. Jones, "Mortality, lifestyle and socio-economic status," Journal of health economics, vol. 27, no. 1, pp. 1-26, 2008.

[22] T. J. Woodruff et al., "Disparities in exposure to air pollution during pregnancy," Environmental Health Perspectives, vol. 111, no. 7, pp. 942, 2003.

[23] P. C. Arden et al., "Cardiovascular mortality and long-term exposure to particulate air pollution epidemiological evidence of general pathophysiological pathways of disease," Circulation, vol. 109, no. 1, pp. 71-77, 2004.

[24] K. Sunil et al., "Lifestyle factors in deteriorating male reproductive health," Indian J Exp Biol, vol. 47, no. 8, pp. 615-24, 2009.

[25] R. V. Bhatt, "Environmental influence on reproductive health," International Journal of Gynecology \& Obstetrics, vol. 70, no. 1, pp. 69-75, 2000.
[26] W. E. Olooto, "Infertility in male; risk factors, causes and management-A review," J. Microbiol. Biotech. Res, vol. 2, no. 4, pp. 641-645, 2012.

[27] Kumar and Sunil, "Occupational exposure associated with reproductive dysfunction," Journal of occupational health, vol. 46, no. 1, pp. 1-19, 2004.

[28] F. Toshiya et al., "Nicotine inhibits pulsatile luteinizing hormone secretion in human males but not in human females, and tolerance to this nicotine effect is lost within one week of quitting smoking," The Journal of Clinical Endocrinology \& Metabolism, vol. 90, no. 7, pp. 3908-3913, 2005.

[29] F. Gladys, "Paternal exposures: impact on reproductive and developmental outcome," An Overview, Pharmacology Biochemistry and Behavior, vol. 55, no. 4, pp. 691-700, 1996.

[30] D.-K. Evanthia et al., "Endocrine-disrupting chemicals: An endocrine society scientific statement," Endocrine reviews, vol. 30, no. 4, pp. 293-342, 2009.

[31] K. Sardar and M. R. Jan, "Assessment of environmental impacts and socio-economic factors of brick kilns in Peshawar, Pakistan," Journal of Himalayan Earth Sciences, p. 33, 2000.

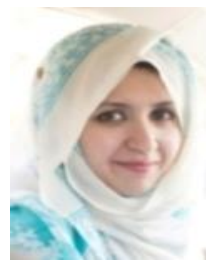

Saadia Shahid was born on Feburary 24 at Wah Cantt. Pakistan. She completed his graduation from Post Graduate College (Wah Cantt). She completed her M.Sc in animal sciences and the M.Phil in reproductive physiology from the Department of Animal Sciences, Quaid-i-Azam University Islamabad. During her research work she developed an interest to work in the field of occupational exposure. Since then she is actively involved in seminars and conferences on occupational health. Her research interests include occupational exposure, International occupational health and health and safety awareness programs. She is also an active member of WHWB which is headed by Marianne Levitsky, a profound researcher. 